\title{
CONSTRAINTS ON GALACTIC INFALL FROM STUDIES OF CHEMICAL EVOLUTION
}

\author{
M.TOSI \\ Osservatorio Astronomico \\ Via Zamboni 33 \\ I-40100 Bologna, Italy
}

ABSTRACT. It is shown that, in order to reproduce the observational properties of our Galaxy, models of galactic chemical evolution require a conspicuous amount of gas falling on the disk with very slow time decay. The constraints on the characteristics of this gas derived from the models are presented.

\section{INTRODUCTION}

The first interpretation of the observational phenomenon of High Velocity Clouds in terms of external gas falling on the galactic disk was given by Oort (1965). A few years later, Larson (1972) computed the first galactic evolution model taking infall explicitely into account, with the aim of preventing a too rapid gas consumption due to the disk star formation. The infalling gas in this case was supposed to originate from the collapsing halo. Since then, infall has become one of the most important but controversial issues in the field of galaxy evolution. It is generally believed that the galactic disk has formed via gas accretion from the halo: what will be discussed here is the existence of gas infall when the disk has already settled in a roughly stable configuration. Several authors consider this infall necessary for a realistic interpretation of the observed galactic properties, whereas others question even its existence or, at least, its relevance in the evolution of our Galaxy.

There are, in fact, some basic open questions about infall which have not received yet a definite answer because of the large uncertainties in the observational data. If any gas actually falls into the disk, what is its amount ? Is the gas of extragalactic origin or is it the residual of the halo collapse ? What are its space and time distributions and its metallicity? We will see in the following that by comparing the observational properties of our Galaxy with the corresponding predictions by models of galactic chemical evolution we can derive useful indications on these issues and reduce the range of possible values of the infall parameters.

\section{MODEL REQUIREMENTS}

The comparison between model predictions and empirical data provided the first information about the infall properties in the early eighties. Tinsley (1980) and 


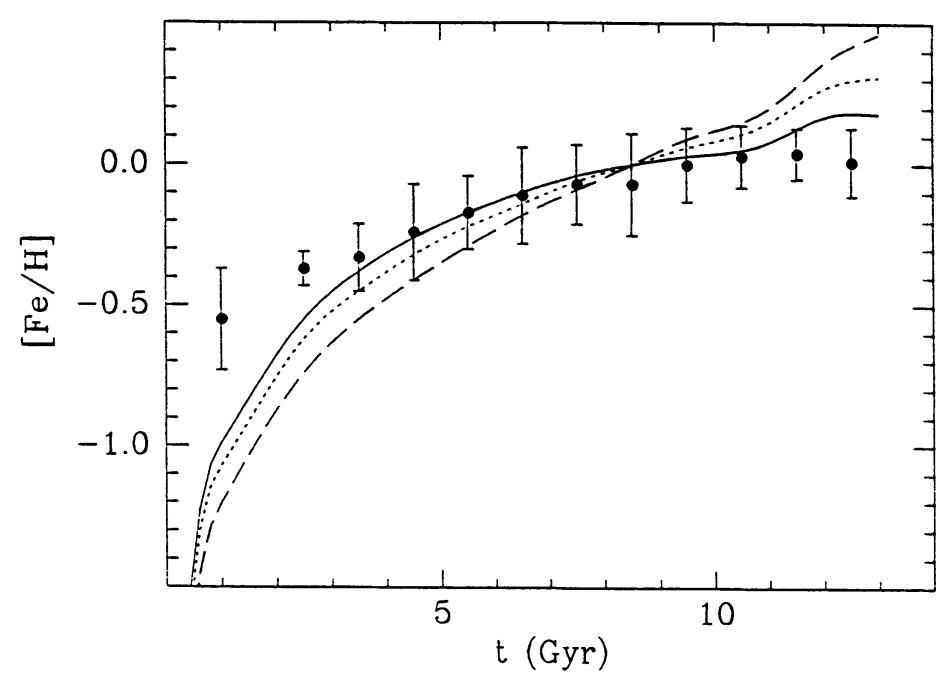

Fig.1. AMR in the solar neighbourhood. The dots with error bars represent Twarog's (1980) data, the dashed line a model with constant SFR and no infall, the dotted line a model with constant SFR and constant infall rate of $410^{-3}$ $\mathrm{M}_{\odot} \mathrm{kpc}^{-2} \mathrm{yr}^{-1}$, the solid line a model with slowly decreasing SFR (e-folding time: $15 \mathrm{Gyr}$ ) and the same infall as the dotted line.

Twarog (1980) showed that to better reproduce the major observational constraints of the solar neighbourhood (age-metallicity relation - AMR - and scarcity of metal poor stars - G-dwarf problem) an infall rate roughly half of the star formation rate - SFR - had to be assumed, and that both rates were approximately constant during the entire Galaxy lifetime. The same conclusions on both infall and SFR were reached by Tosi (1982) to reproduce the abundance gradients and the gas and total mass distributions observed in the disk.

Figure 1 shows the AMR derived by Twarog from a sample of $\sim 1000 \mathrm{~F}$ stars in the solar neighbourhood. The error bars correspond to his estimated uncertainties on the data. The dashed curve represents a model with constant SFR and no infall after the disk formation: it is apparent that it predicts too low abundances in the early epochs and too large abundances at the present time. A model with the same SFR and constant infall (dotted curve) is more consistent with the data because the lower initial gas content of the region is more readily polluted by the metals ejected by evolving stars, thus allowing a faster rise of the AMR curve in the early epochs and because the infall of metal poor gas dilutes the chemical abundance of the interstellar medium in the recent phases. A model with the same infall and slowly decreasing SFR is in better agreement with the data because it provides more star (and metal) formation in the early epochs and less in the late epochs than the previous two models. Had we assumed for the initial disk metallicity a finite value instead of $Z=0$, we would have obtained a good fit also for the left part of the diagram (see e.g. Matteucci and François 1989). 
Most chemical evolution models do not consider at all the dynamics of the system. Hence, the effect of infall simply corresponds to an increase of the gas reservoir and, since the infalling gas is usually assumed to be metal poor, its major result is to dilute the ambient metallicity. However, an important caveat for models with infall was pointed out by Mayor and Vigroux (1981), who showed that the reduction of angular momentum suffered by the infalling gas when reaching the disk causes an inevitable radial (inward or outward) motion of this gas. Radial flows must then be taken into account if infall is assumed. Models taking radial flows into account have later been computed by Lacey and Fall (1985), Tosi (1988a) and Pitts and Tayler (1990). Despite the different approaches and approximations, they all found that inward radial flows steepen the metallicity gradients but that infall is still required to reproduce all the galactic properties even when radial flows are included in the models.

During the last ten years, there have been some attempts to demonstrate that infall is not necessary to reproduce the galactic properties, if particular choices of the other parameters (SFR, initial mass function and stellar parameters, see Tosi 1988a) are adopted. For instance, Gusten and Mezger (1982) proposed as an alternative solution a bimodal SFR with only stars more massive than $3 \mathrm{M}_{\odot}$ forming on spiral arms. Rana and Wilkinson $(1986,1988)$, on the other hand, suggested that the SFR be proportional only to the molecular hydrogen density, rather than to the total gas density or mass. These proposed SFRs share the property of being larger in the inner than in the outer galactic regions, thus producing easily the radial abundance gradients. However, up to now the only models in satisfactory agreement with all the observational constraints of our Galaxy are those assuming a conspicuous, long lasting infall (Lacey and Fall 1985, Tosi 1988a, Matteucci and François 1989, Pagel 1989). For instance, Figure 2 shows some of the results derived for the Galaxy from recent calculations by Tosi and Diaz (1990). The top left panel represents the current radial distribution of the SFR as derived from Lacey and Fall's collection of observational data available in the literature. The top right panel represents the radial distribution of the molecular hydrogen density given by Talbot (1980), and the bottom panel the oxygen abundance gradient derived by Shaver et al. (1983) from optical and radio observations of HII regions. The solid line in all panels corresponds to a model with very slowly decreasing SFR (e-folding time: $15 \mathrm{Gyr}$ ) and gas falling on the disk at a constant rate and with uniform density (hereinafter: reference model). All the other curves refer to models $\dot{a}$ la Rana and Wilkinson, i.e. with SFR proportional to the molecular hydrogen density through a metallicity-molecular hydrogen relation (see Tosi and Diaz 1990, for details). All the models reproduce fairly well the observed SFR and oxygen distributions, but the infall model is significantly better than the others as for reproducing the molecular hydrogen data. It seems inevitable to reject models which assume a SFR proportional to molecular hydrogen but are unable to reproduce its observed distribution!

By focusing our attention on the oxygen abundance distribution, we can get some more detailed information on the infall requirements. Figure 3 shows again Shaver's et al. data and the reference model of the previous graphs (bottom solid 

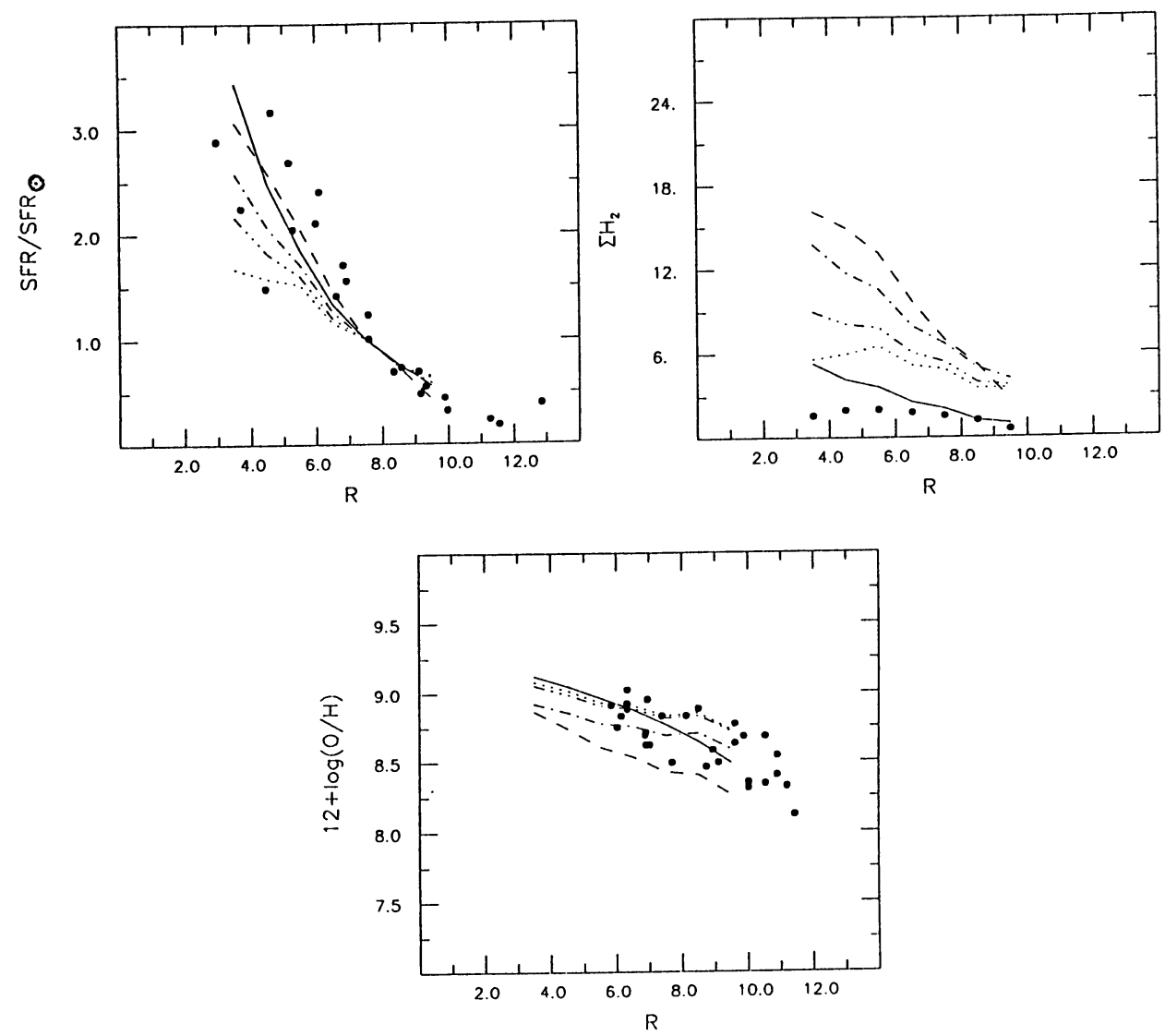

Fig.2. Comparison between observed and predicted radial distribution of the SFR (top left), the molecular hydrogen density (top right) and oxygen abundance (bottom). The solid line represents the reference model, the others refer to models $\dot{a}$ la Rana and Wilkinson.

line). The dotted line corresponds to a similar model with more rapidly decreasing $\mathrm{SF}$ and infall rates and gives also a good fit to the data. If we keep unchanged all the parameters adopted for the reference model but assume no infall, the resulting oxygen abundances are given by the dash-two-dotted line: clearly too flat and too metal rich with respect to the observations, due to the lack of diluting mechanisms for the metals synthesized by the stars. If we keep unchanged all the parameters, reinclude infall, but assume that its density instead of being uniform increases inwards (for instance as a function of the total mass of the region) we obtain the dash-dotted line of Fig.3. The flatness of this curve is due to the fact that the larger metal production of the inner galactic regions is now overcompensated by their larger infall rate, and is inconsistent with the observed gradient. Finally, the dashed 


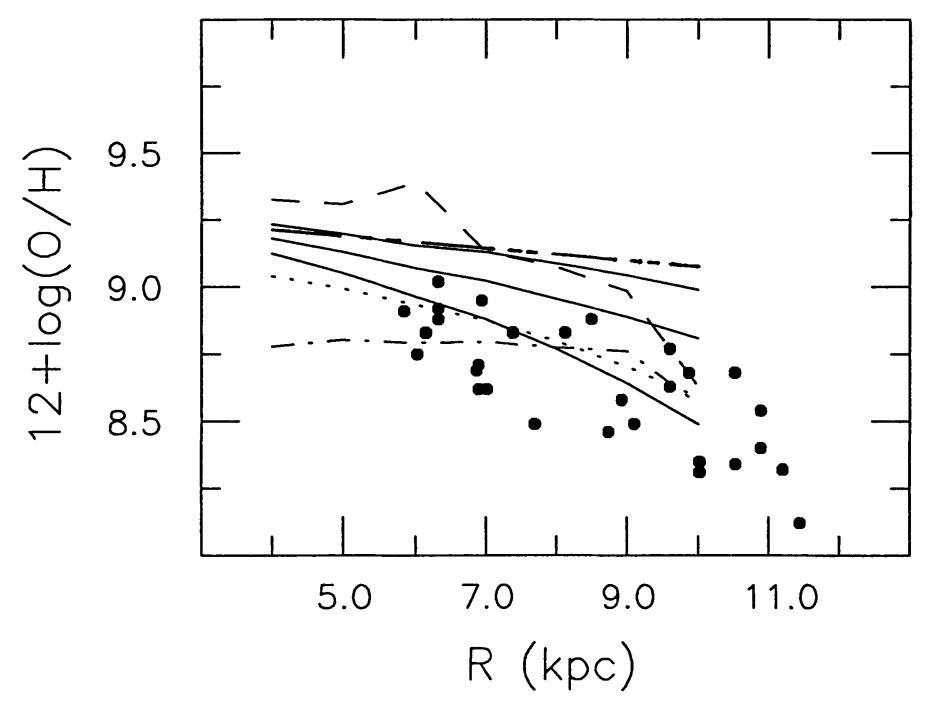

Fig.3. Oxygen abundance distribution in the galactic disk as derived from HII region observations (dots) and model predictions. The solid lines represent the reference model (see text) with increasing infall metallicity from bottom to top. The dash-dotted line corresponds to the same model as the bottom solid line, but with infall density increasing inwards; the dash-two-dotted line refers to the same model, but without infall. The dotted line corresponds to a model similar to that of the solid line but with different e-folding times of the SFR and the infall rate, and the dashed line to a model $\dot{a}$ l $a$ Gusten and Mezger.

line represents a model $a$ a $l a$ Gusten and Mezger. Since oxygen is mostly produced by massive stars, their larger fraction on spiral arms produces the bump shown in the Figure in correspondence with the Sagittarius arm. Again the morphology of the resulting oxygen distribution and its large abundances are inconsistent with the observed data.

The same set of data provides information also on the infall metallicity. Till now, the infalling gas has been assumed to be primordial (i.e. to contain no metals), but this assumption is perhaps unrealistic not only in the case of halo gas, which should have typical Population II abundances, but even in the case of extragalactic gas, which may have been polluted through galactic winds. If we consider in Figure 3 the reference model represented by the bottom solid line, leave all the parameters unchanged but consider the infall metallicity $\mathrm{Z}_{f}$ to be as large as $0.1 \mathrm{Z}_{\odot}$ and the various element abundances to be in solar proportions, the resulting line is in practice undistiguishable from the reference line. For increasing $\mathrm{Z}_{f}$, the metal dilution by the infall gas is less effective and the resulting oxygen abundances become larger 
and larger and their gradient increasingly flat. We can see from Fig.3 that the middle solid line (corresponding to $\mathrm{Z}_{f}=0.5 \mathrm{Z}_{\odot}$ ) is already at the upper limit of the observed oxygen distribution and the top solid line $\left(\mathrm{Z}_{f}=\mathrm{Z}_{\odot}\right)$ is definitely inconsistent with the data. Equivalent results are obtained also for the other models (Tosi $1988 b)$.

To generalize as much as possible the model constraints on galactic infall, we have computed a large number of models with very different assumptions about the major parameters and compared their results with the largest number of observed galactic properties (Tosi 1988a) available in the literature. The $\chi^{2}$ resulting from the comparison of each independent set of observational constraints with the corresponding model predictions has been computed. For each class of models (i.e. models where all the other parameters were fixed except the infall rate density $F$ and e-folding time $\theta$ ), the combination of infall parameters leading to the minimum $\chi^{2}$ in the comparison with each of the observational constraints was found. In the F- $\log \theta$ plane of Figure 4, the projection of the regions defined by $\chi^{2}<\chi_{\min }^{2}+4$ on each axis yields the $95 \%$ confidence limit on the infall rate density and e-folding time parameters (Avni 1976). An example of the method is given in Fig.4 for models with exponentially decreasing SFR (e-folding time: $15 \mathrm{Gyr}$ ) and exponentially decreasing infall rate with uniform density. For sake of simplicity, Fig.4 shows only the surfaces relative to the stellar metallicity distribution with galactocentric distance (dotted curves), the oxygen abundance gradient derived from HII regions (dashed curves) and the AMR derived by Twarog (1980) for the solar neighbourhood (solid curves). The $\chi^{2}$, however, has been computed also for the gas and total mass distributions, the radial profile of the current SFR and the gradients in the abundances of other elements. Only the combination of parameters falling in the intersecting portion of all the surfaces provides results simultaneously consistent with the three sets of data. The shaded areas correspond to forbidden combinations of the infall parameters leading to unphysical conditions (e.g. negative initial mass of the disk or too rapid gas consumption). The left panel refers to models without radial flows and the acceptable values of infall parameters in this case are $\mathrm{F} \sim 410^{-3}$ $\mathrm{M}_{\odot} \mathrm{kpc}^{-2} \mathrm{yr}^{-1}$ and $\theta \geq 100 \mathrm{Gyr}$ (i.e. almost constant rate). The right panel refers to models where all the infallen gas is forced to move inward. No intersection exist in this case, thus showing that this kind of extreme models do not apply to our Galaxy and that radial flows should be more moderate. Note that in any case the surfaces corresponding to a good agreement between data and predictions lie at large $\theta$ : in practice, the time decay of the infall rate must have been quite slow.

The results of applying the $\chi^{2}$ method to a significantly large sample of models with very different assumptions on the various parameters and the arguments presented above can be schematically summarized with the following model constraints on galactic infall:

a) the current infall rate ranges between 0.3 and $1.8 \mathrm{M}_{\odot} \mathrm{yr}^{-1}$ for the whole disk;

b) its e-folding time should be longer than that of the SFR. Since the SFR has decreased quite slowly with time (e-folding time $\geq 5 \mathrm{Gyr}$ ), this latter result implies that the infall rate must have been almost constant too; 


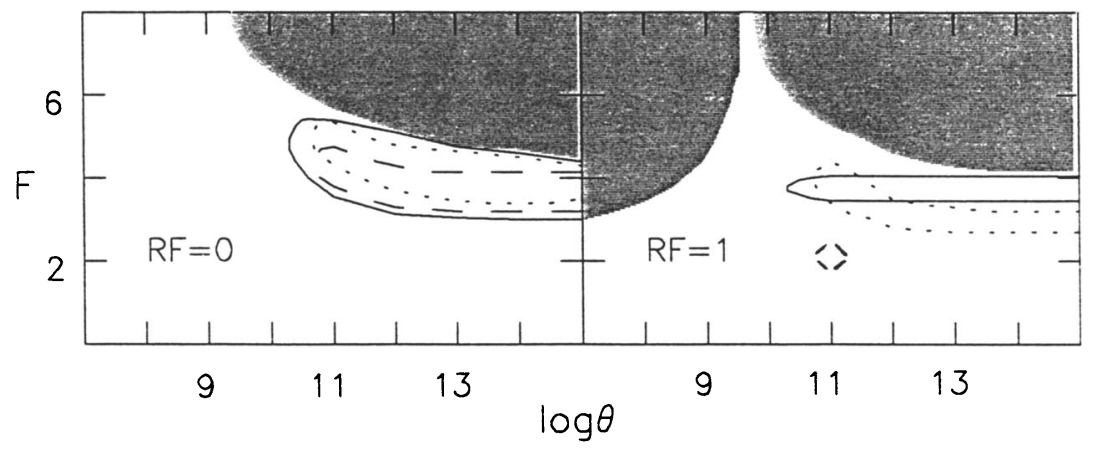

Fig.4. Contours of $95 \%$ confidence level for models with exponentially decreasing SFR, exponentially decreasing infall and uniform infall density F. RF indicates the amount of radial flows normalized to the infalling gas. The contours correspond to the AMR (solid curves), radial distribution of the stellar metallicity (dotted curves) and of the oxygen abundance of HII regions (dashed curves). F is in units of $10^{-3}$ $\mathrm{M}_{\odot} \mathrm{kpc}^{-2} \mathrm{yr}^{-1}$ and $\theta$ in Gyr.

c) the infall rate density $\left(\mathrm{M}_{\odot} \mathrm{kpc}^{-2} \mathrm{yr}^{-1}\right)$ must have been uniform or increasing outwards, certainly not inwards;

d) the required diluting effect is consistent with infall metallicities up to $0.3 \mathrm{Z}_{\odot}$.

\section{DISCUSSION}

It is important to emphasize that items $a) \rightarrow d$ ) of the previous section, do not provide any clue to understand the origin of the infalling gas. Both an intergalactic gas and a residual of a slow halo collapse can fulfill all the above requirements. Very recent calculations (Matteucci and François 1989, Pagel 1989, Ferrini et al. 1990) have modeled together the disk and halo phases of the Galaxy evolution and, despite the different approaches, derive infall properties very similar to those described above. They all suggest that the initial rapid collapse of the halo gas on the disk is followed by a slow gas capture by the disk. However, the suggested source of this captured gas varies from one author to another: some consider it as pure halo gas, some as a mixture of halo and thick disk gas, others as a mixture of halo and extragalactic gas.

The situation in other galaxies is even more uncertain. The paucity of accurate and extensive observational data reduces the reliability of the chemical evolution models. For instance, there are only eight nearby spirals for which the gas and total mass distributions are well defined and the oxygen abundances accurately derived from HII region observations. For these galaxies Tosi and Diaz (1985) have computed models of the type of the reference model described above. What we generally found is that an infall of the same kind as that appropriate for our 
Galaxy appears to be needed to dilute the metallicity of more massive galaxies, whilst in smaller objects it does not seem to be necessary. Perhaps for these lower mass spirals galactic winds would improve the agreement between predictions and empirical data, as already suggested for irregular galaxies (Matteucci and Tosi 1985).

The consistency of our derived infall properties with the current observational scenario of High and Very High Velocity Clouds (HVCs and VHVCs) is not completely settled. There seems to be a general agreement on an overall infall rate of $0.1 \mathrm{M}_{\odot} \mathrm{yr}^{-1}$ from VHVCs. However, the controversy over the interpretation of HVCs (e.g. Mirabel 1981, Mirabel and Morras 1984, Danly 1989, Wakker 1990) and the corresponding current infall rate $\left(0.1\right.$ or $1 \mathrm{M}_{\odot} \mathrm{yr}^{-1}$ ?) does not allow yet to understand if our theoretical estimates are in agreement with the observational evidence. Certainly, up to now no model of galactic chemical evolution with current infall rate as small as $0.1 \mathrm{M}_{\odot} \mathrm{yr}^{-1}$ has been successful in reproducing all the Galaxy properties.

As for the metallicity, instead, the observational estimates (e.g. De Boer and Savage 1983, 1984) are consistent with our upper limit of $\mathrm{Z}_{f} \leq 0.3 \mathrm{Z}_{\odot}$. Since both the halo and the intergalactic gas have apparently an average metallicity lower than this limit, our model results cannot discriminate between these two possible origins of infall. Viceversa, galactic fountains can be excluded as its major sources (but they can be present if some other source of metal poor gas is found). Their metal content, in fact, will mostly be that expelled by Supernovae, much higher and enhanced in some particular elements (e.g. oxygen for $\mathrm{SNe} I I$ and iron for $\mathrm{SNe}$ I) than the average interstellar medium. The diluting effect of infall in this case would be completely lost.

It is of fundamental importance that the big effort currently made by several observers to reach more accurate information find soon a satisfactory achievement. The major points that require an observational answer are the following: a) what are the clouds (HVCs or only VHVCs ?) that can safely be taken as evidence of gas infall, b) what current infall rate do they imply, c) what is their metallicity and the relative proportions (solar or not ?) of the various elements, d) are the features of the gas recently discovered around other spirals consistent with those of an infall ?

\section{REFERENCES}

Avni, Y. 1976, Astrophys.J. 210, 642

Danly, L. 1989, Astrophys.J. 342, 785

De Boer, K.S. and Savage, B.D. 1983, Astrophys.J. 265, 210

De Boer, K.S. and Savage, B.D. 1984, Astr.Astrophys.Letters 136, L7

Ferrini, F., Matteucci, F., Pardi, C., Penco, U. 1990, in preparation

Gusten, R. and Mezger, P.G. 1982, Vistas Astron. 26, 159

Lacey, C.G. and Fall, S.M. 1985, Astrophys.J. 290, 154

Larson, R.B. 1972, Nature 236, 21

Matteucci, F. and François, P. 1989, Mon.Not.Roy.Astr.Soc. 239, 885

Matteucci, F. and Tosi, M. 1985, Mon.Not.Roy.Astr.Soc. 217, 391

Mayor, M. and Vigroux, L. 1981, Astr.Astrophys. 98, 1 
Mirabel, I.F. 1981, Rev.Mex.Astron.Astrophys. 6, 245

Mirabel, I.F. and Morras, R. 1984, Astrophys.J. 279, 86

Oort, J.M. 1965, Trans.IAU 12A, 789

Pagel, B.E.J. 1989, Rev.Mex.Astron.Astrofis. in press

Pitts, E. and Tayler, R.J. 1990, Mon.Not.Roy.Astr.Soc. 240, 373

Rana, N.C. and Wilkinson, D.A. 1986, Mon.Not.Roy.Astr.Soc. 218, 497

Rana, N.C. and Wilkinson, D.A. 1988, Mon.Not.Roy.Astr.Soc. 231, 509

Shaver, P.A., McGee, R.X., Newton, L.M., Danks, A.C. and Pottasch, S.R. 1983, Mon.Not.Roy.Astr.Soc. 204, 53

Talbot, R.J.Jr. 1980, Astrophys.J. 235, 821

Tinsley, B.M. 1980, Fund.Cosmic Phys. 5, 287

Tosi, M. 1982, Astrophys.J. 254, 699

Tosi, M. 1988a, Astron.Astrophys. 197, 33

Tosi, M. 1988b, Astron.Astrophys. 197, 47

Tosi, M. and Diaz, A.I. 1985, Mon.Not.Roy.Astr.Soc. 217, 571

Tosi, M. and Diaz, A.I. 1990, Mon.Not.Roy.Astr.Soc. in press

Twarog, B.A. 1980, Astrophys.J. 242, 242

Wakker, B.P. 1990, this conference 


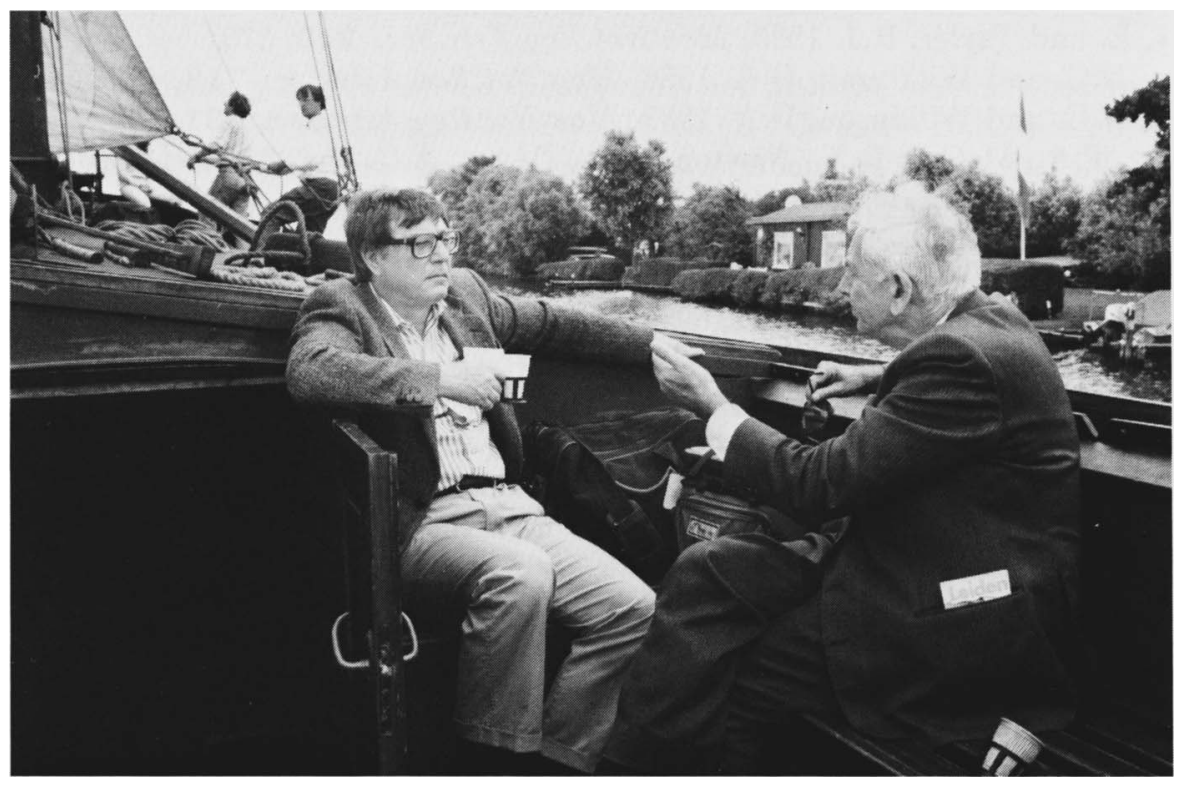

